\title{
CLADISTICS AND RATES OF MORPHOLOGICAL EVOLUTION: COMPUTATION AND COMPARISON
}

CLOUTIER*, Richard, Dept. of Palaeontology, The Natural History Museum, Cromwell Rd., London, SW7 5BD, U.K.; ROWE, Timothy, Dept. of Geological Sciences, University of Texas, Austin, TX, 78713, U.S.A.

For the part fifty years, the concept of evolutionary rates has been developed largely in an evolutionary or phenetic framework. Many authors discussed rates, but under previous systematic paradigms no standardized methods developed to provide a uniform and general framework in which temporal properties of different lineages could be objectively measured and compared.

We review recently developed methods for measuring rate-related properties of lineages that are based on phylogenetic analyses. Rate measurements are made directly from phylogenetic data matrices and cladograms. Because they benefit from the standardized procedures required to compile a data matrix, these methods are potentially exportable to a wide range of phylogenetic studies. Four potential factors relevant to the study of evolutionary rates in a cladistic framework have been analyzed with these methods: (1) morphological changes, (2) age and duration, (3) cladogenesis, and (4) species-diversity. We investigate interactions among these factors.

Underlying assumptions in using a cladogram to calculate rates of evolution are: (1) the tree reflects the history of the group, and (2) the distributions of character states on the tree reflects the true distribution of character states during the evolution of the group. The calculation of rates from a cladogram requires that: (1) the cladograms are derived from parsimony analysis (e.g., PAUP, HENNIG86) of species coded for discrete character states; (2) the phylogenetic pattern (or branching sequence) is superimposed on a geological time scale in which each species is mapped according to its geological age (temporal cladogram); (3) phylogenetic pathways (unidirectional series of consecutive cladogenetic events) are determined in such a manner that the selected origin is a hypothetical ancestor to the terminal taxa selected; and (4) rates are calculated along selected phylogenetic pathways.

Rates may be calculated as the number of changes-including autapomorphies, synapomorphies, and homoplasies-per unit of time. Alternatively, relative measures of rate may be obtained by comparing the average number of changes for an entire data matrix with changes in data subsets within the matrix. Five types of rates of morphological evolution can be determined and quantified along a phylogenetic pathway: (1) rate between two consecutive cladogenetic events, (2) rate during a geological period, (3) rate during a fixed period of time in millions of years, (4) relative rates of change for different morphological data subsets, such as dentition versus skeleton, and (5) relative rate along a selected segment of the cladogram, compared to the average rate measured for the cladogram as a whole. Various methods for comparing rates are investigated, such as Spearman Rank correlation, Fourier analysis, time series, and homoplasy index.

Classic examples of rates of morphological evolution were previously taken from bivalves, echinoderms, lungfishes, coelacanths, and mammals. In the present study, rates of evolution within and/or between the Dipnoi (lungfishes), the Actinistia (coelacanths), and the Mammalia are calculated and compared. 